\title{
Digitale Rentner/-innen?
}

\section{Internetnutzung ab 65 Jahren in der Schweiz}

\author{
Alexander Seifert \& Hans Rudolf Schelling
}

\begin{abstract}
Das Internet gewinnt immer mehr an Bedeutung im Alltag, dennoch nutzen ältere Menschen dieses Medium weniger als jüngere. Eine Schweizer Studie untersuchte die Internetnutzung bzw. -nichtnutzung von Personen ab 65 Jahren.
\end{abstract}

\section{Des retraités numériques ? Utilisation d'Internet à partir de 65 ans en Suisse \\ L'Internet prend de plus en plus d'importance au quotidien et pourtant les personnes âgées utilisent moins ce média que les jeunes. Une étude suisse a examiné l'utilisation ou la non-utilisation d'Internet par les personnes de plus de 65 ans.}

DOI 10.1024/2297-5160/a000010

I nformationen und Kommunikationsangebote konzentrieren sich immer mehr auf neue Medien wie das Internet. Während die Altersgruppen bis 64 Jahre seit den 1990er Jahren einen starken Anstieg in der Internetnutzung aufweisen, bleibt die intensive Nutzung der Altersgruppen ab 65 Jahren zurück. Mit dem Interesse, mehr über Hemmnisse und Anreize der Internetnutzung bei älteren Menschen zu erfahren, initiierte Pro Senectute Schweiz 2009 und 2014 jeweils eine Studie. Mittels einer repräsentativen telefonischen und postalischen Erhebung in der gesamten Schweiz wurden in der aktuellen Erhebung (2014) bei insgesamt 1037 älteren Personen ab 65 Jahren Informationen zur Person, ihrer Techniknutzung und -einstellung, ihrem Internetnutzungsverhalten und ihrer Einstellung zum Internet erhoben. Es konnten sowohl Personen befragt werden, die das Internet nutzen («Onliner»), als auch Personen, die das Internet nicht nutzen («Offliner»).

\section{Die Internetnutzung nimmt zu, doch nutzen mehr als $\mathbf{4 0} \%$ das Internet nicht}

Der direkte Vergleich mit der 2009 durchgeführten ersten Befragung zeigt, dass sich die Internetnutzung in den letzten fünf Jahren auch bei der Bevölkerungsgruppe der

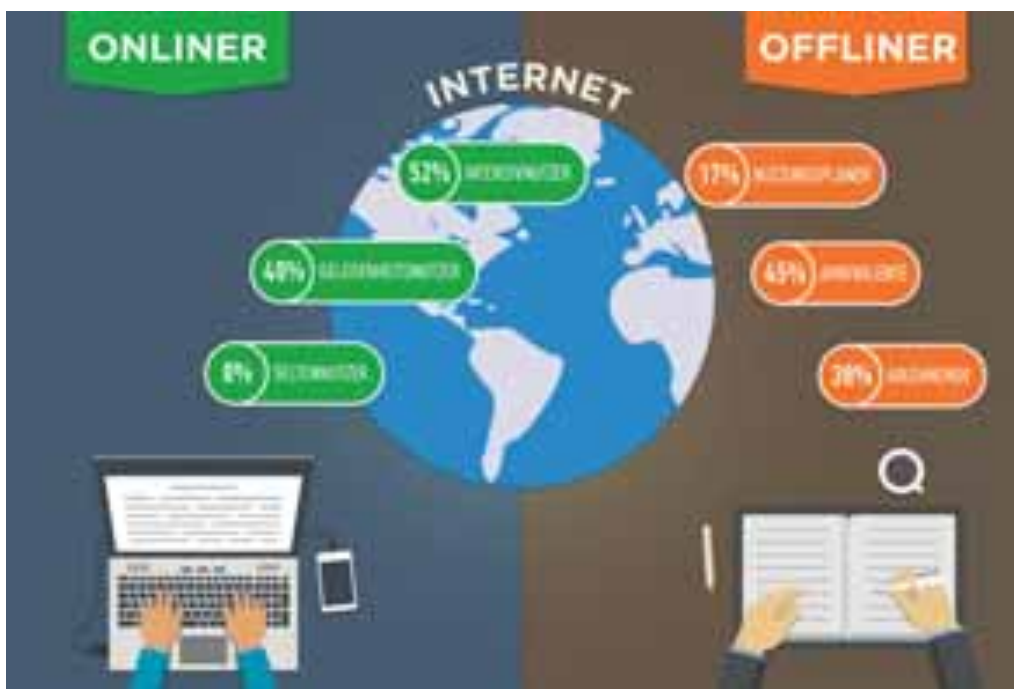

(C) Alexander Seifert

65-Jährigen und Älteren weiter verbreitet hat. Waren es 2009 noch $38 \%$ der Befragten, die angaben, das Internet zu nutzen, sind es nun $56 \%$. Dennoch ist auch weiterhin eine «digitale Spaltung» zwischen den Generationen der unter und über 65-Jährigen festzustellen. Diese Nutzungsunterschiede zeigen sich auch zwischen den Altersgruppen der Älteren selbst; so nutzen Personen ab 80 Jahren das Internet deutlich seltener als Personen im Alter zwischen 65 und 75 Jahren. Während es nur 13\% Onliner unter den ab 85-Jährigen gibt, sind es bei den befragten 65bis 69-Jährigen immerhin $79 \%$. Frauen sind $\mathrm{zu} 46 \%$ Onliner, Männer zu 68\%. Befragte Personen, die das Internet nutzen, tun dies zu 52\% täglich, zu 31\% mehrmals pro Woche und zu $17 \%$ seltener. Die Nutzung erfolgt mehrheitlich zu Hause, jedoch gibt ein Drittel der Onliner an, das Internet auch unterwegs (mit Smartphone oder Tablet-Computer) zu nutzen.

\section{Wie unterscheiden sich Onliner von Offlinern?}

Die Gruppen der On- und Offliner sind jeweils sehr heterogen. Es können jeweils drei Gruppen unterschieden werden: 
- Onliner:Intensivnutzer/Gelegenheitsnutzer/Seltennutzer

- Offliner: Nutzungsplaner/Ambivalente/Ablehnende

Das kalendarische Alter allein kann die Nichtnutzung des Internets nicht erklären, auch wenn das Alter einen hohen statistischen Erklärungswert besitzt. Vielmehr profitiert die Nutzung des Internets sowohl von sozioökonomischen als auch von gesundheitlichen und sozialen Ressourcen. Neben Ressourcen im engeren Sinn bestimmen auch die persönliche Technikaffinität und -bewertung das tatsächliche Nutzungsverhalten. Die Wahrscheinlichkeit einer Internetnutzung ist also höher, wenn hinreichende Ressourcen (Bildung, Einkommen, Gesundheit, Technikkompetenz, Zuraten aus dem sozialen Umfeld) vorhanden sind und wenn eine Affinität zu Technik sowie eine positive Einstellung zum Internet und $\mathrm{zu}$ dessen Nutzung - im Sinne von «die Nutzung ist nützlich und leicht»-bestehen.

\section{Anwendungsbereiche und Hemmnisse}

Die tatsächliche Nutzung der Onliner und die Nutzungsinteressen der Offliner beziehen sich auf ähnliche Anwendungen. Bevorzugt werden allgemeine Funktionen wie E-Mails schreiben, Informationen suchen oder Fahrpläne abfragen. Weniger genutzt beziehungsweise weniger interessant sind spezifische Anwendungen wie Multimediainhalte, soziale Netzwerke oder der Verkauf von Waren. Von Onlinern wurden als aktuelle Schwierigkeiten bei der Nutzung des Internets besonders häufig genannt: Sicherheitsbedenken (56\%), Angst vor technischen Problemen (24\%) und geringe Glaubwürdigkeit von Informationen (23\%). Am häufigsten gaben Offliner diese Gründe für die Nichtnutzung an: Kompliziertheit der Benutzung (70\%), Sicherheitsbedenken (64\%) und zu hoher Aufwand beim Erlernen (63\%).

\section{Die mobile Nutzung des Internets}

Innerhalb der aktuellen Befragungsstudie konnten erstmals auch die Nutzung von Smartphones und Tablet-Computern sowie die Verwendung des mobilen Internets erforscht werden. Es zeigte sich, dass etwa ein Drittel der befragten Personen ein solches mobiles Gerät besitzt und dieses häufig verwendet - auch um damit unterwegs «ins
Internet zu gehen». Bereits $32 \%$ der befragten Personen ab 65 Jahren verfügen über ein internetfähiges Smartphone und $26 \%$ über einen Tablet-Computer in ihrem Haushalt. Personen, die das mobile Internet nutzen, gehören hauptsächlich der Gruppe der «Intensivnutzer» an; sie nutzen nicht nur den klassischen Computer fast täglich, sondern auch mobile Endgeräte, um damit ins Internet zu gehen.

\section{Das Gefühl der Ausgeschlossenheit als Offliner}

$14 \%$ der Offliner fühlen sich aus der Gesellschaft ausgeschlossen, weil sie das Internet nicht nutzen; sogar 33\% der Onliner würden sich ausgeschlossen fühlen, wenn sie das Internet nicht mehr nutzen könnten. In der Selbstwahrnehmung eines Teils der befragten Personen ist die Gefahr eines Ausschlusses präsent - und zwar vermehrt bei Onlinern (insbesondere Intensivnutzern), für die der Verlust des Internetzugangs einen Einschnitt in ihre heutigen Möglichkeiten bedeuten würde.

\section{Schlussbemerkungen}

Die Digitalisierung des Alltags wird weiter voranschreiten und damit auch die Bedeutung des Internets im Alltag älterer Menschen. Auch wenn sich die reine Nutzungshäufigkeit zwischen den Generationen in den nächsten Jahren immer mehr angleichen mag, wird das Thema «Techniknutzung im Alter» durch immer wieder neue Technologien nicht an Aktualität verlieren. Grundsätzlich ist eine individuell gewollte Nichtnutzung des Internets zu akzeptieren; gleichzeitig ist zu vermeiden, dass weniger technikaffine Personen aus der Gesellschaft (und von Informationen) ausgeschlossen werden.

Informationen zur Studie:

http://www.zfg.uzh.ch/projekt/ikt-alter-2014.html

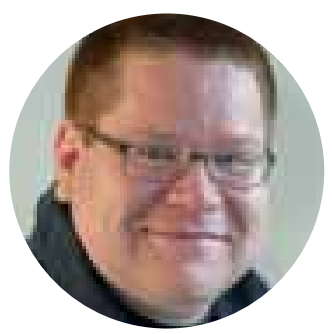

Alexander Seifert arbeitet als wissenschaftlicher Mitarbeiter am Zentrum für Gerontologie der Universität Zürich.

alexander.seifert@zfg.uzh.ch 